\title{
On a generalization of the Cartwright-Littlewood fixed point theorem for planar homeomorphisms
}

\author{
J. P. BOROŃSKI \\ National Supercomputing Center IT4Innovations, Division of the University of Ostrava, \\ Institute for Research and Applications of Fuzzy Modeling, \\ 30. dubna 22, 70103 Ostrava, Czech Republic \\ Faculty of Applied Mathematics, AGH University of Science and Technology, \\ al. Mickiewicza 30, 30-059 Kraków, Poland \\ (e-mail: jan.boronski@osu.cz)
}

(Received 18 June 2014 and accepted in revised form 22 October 2015)

Abstract. We prove a generalization of the fixed point theorem of Cartwright and Littlewood. Namely, suppose that $h: \mathbb{R}^{2} \rightarrow \mathbb{R}^{2}$ is an orientation preserving planar homeomorphism, and let $C$ be a continuum such that $h^{-1}(C) \cup C$ is acyclic. If there is a $c \in C$ such that $\left\{h^{-i}(c): i \in \mathbb{N}\right\} \subseteq C$, or $\left\{h^{i}(c): i \in \mathbb{N}\right\} \subseteq C$, then $C$ also contains a fixed point of $h$. Our approach is based on Brown's short proof of the result of Cartwright and Littlewood. In addition, making use of a linked periodic orbits theorem of Bonino, we also prove a counterpart of the aforementioned result for orientation reversing homeomorphisms, that guarantees a 2-periodic orbit in $C$ if it contains a $k$-periodic orbit $(k>1)$.

\section{Introduction}

In 1951 Cartwright and Littlewood [14] studied van der Pol's differential equation and were led to investigate the existence of fixed points of planar homeomorphisms in invariant continua. The continuum that they encountered had a boundary that was not locally connected and was potentially even indecomposable. Recall that a continuum is a connected and compact non-degenerate set. It is indecomposable if it is not the union of any two proper subcontinua. A planar continuum is acyclic if it does not separate the plane. The following is the celebrated Cartwright-Littlewood fixed point theorem.

THEOREM A. [14] Let $f: \mathbb{R}^{2} \rightarrow \mathbb{R}^{2}$ be an orientation preserving planar homeomorphism. Suppose that there is an acyclic continuum $C$ invariant under $f$; i.e. $f(C)=C$. Then there is a fixed point of $f$ in $C$. 


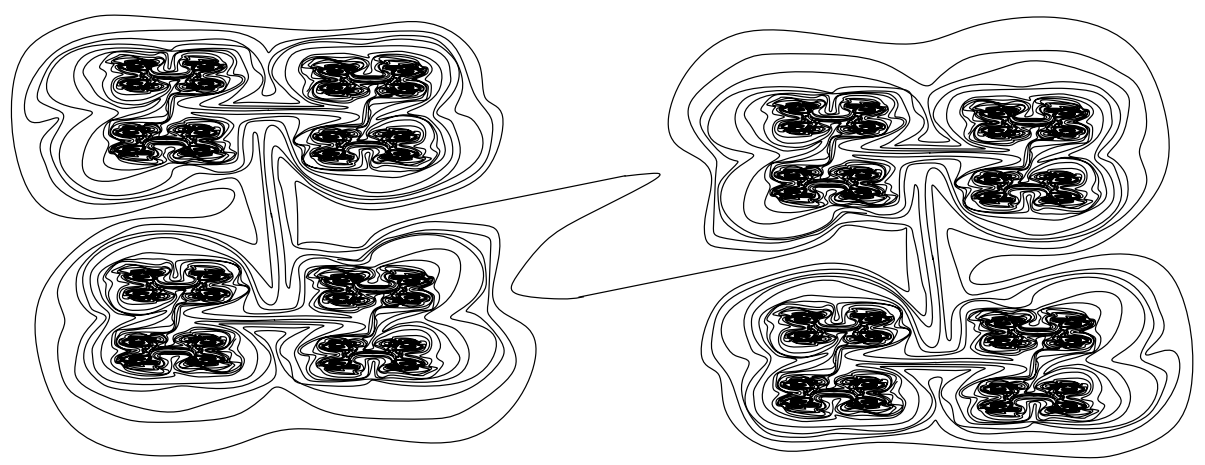

FIGURE 1. An (acyclic) arc-like chaotic attractor without indecomposable subcontinua.

A number of authors provided alternative proofs of the result, many of which are substantially shorter then the original one, including those given by Brown [13] and Hamilton [15]. Barge and Gillette proved that the continuum considered by Cartwright and Littlewood was in fact indecomposable [4]. Barge and Martin also showed [3] that any inverse limit of arcs with a single continuous bonding map gives an acyclic continuum (called arc-like $\dagger$ ) that is an attractor of a planar homeomorphism. The class of arc-like continua includes the famous hereditarily indecomposable pseudo-arc [6] (which is homogeneous, contains no arcs and is nowhere locally connected) and Knaster buckethandle continuum [1] (cf. topological horseshoe [21]). There is often a strong connection between the topology of the attracting continua and the complexity of the associated dynamics, like the link between indecomposability and chaos [2] (a chaotic decomposable arc-like attractor of a planar homeomorphism without indecomposable subcontinua recently constructed by Oprocha and the author [10] is depicted in Figure 1). In addition, Pliss in [20] showed that any acyclic plane continuum is the maximal bounded closed set invariant under a transformation $F$, where $F$ is a solution of certain dissipative system of differential equations. Note that Theorem A holds true for orientation reversing homeomorphisms, by the result of Bell [5], and Kuperberg generalized Bell's result to plane separating continua $[\mathbf{1 6}, \mathbf{1 7}]$. However, it remains a major 100 -year-old open problem whether every acyclic planar continuum has the fixed point property (see Scottish Book Problem 107 [18], and [7] for the most recent developments regarding the subject).

In the present paper we are interested in generalizing Theorem A in the following direction. Suppose that a continuum $C$ is not necessarily invariant under $h$. Can one find some natural conditions under which a component of $C \cap h(C)$ contains a fixed point? Finding such conditions has the potential to give a lower bound for the number of fixed points of $h$, which could prove very useful if $C \cap h(C)$ has more than just one component. Such conditions could also aid in locating fixed points of $h$. With this goal in mind, we shall demonstrate that, given a continuum $C$, in order for $h$ to have a fixed point in $C$ it suffices that $h^{-1}(C) \cup C$ is acyclic and there is a backward or forward orbit $\mathcal{O}$ entirely contained in $C$. As a consequence we get that if $h$ has no fixed point in $C$ then $C$ also does not contain

$\dagger$ A continuum is called arc-like, chainable, or snakelike if it can be given as the inverse limit of arcs with continuous bonding maps. 
any periodic orbits. A related result has recently been obtained by Ostrovski [19], with very strong assumptions on the topological structure of $C$. Namely, by entirely different methods, Ostrovski proved the following two theorems.

THEOREM B. [19] Let $X \subset \mathbb{R}^{2}$ be a compact, simply connected, locally connected subset of the real plane and let $f: X \rightarrow Y \subset \mathbb{R}^{2}$ be a homeomorphism isotopic to the identity on $X$. Let $C$ be a connected component of $X \cap Y$. If $f$ has a periodic orbit in $C$, then $f$ also has a fixed point in $C$.

THEOREM C. [19] Let $D \subset \mathbb{R}^{2}$ be a Jordan domain and $f: D \rightarrow E \subset \mathbb{R}^{2}$ an orientation preserving homeomorphism. Let $C$ be a connected component of $D \cap E$. If $f$ has a periodic orbit in $C$, then $f$ also has a fixed point in $C$.

Theorem $\mathrm{C}$ is in fact a corollary to Theorem $\mathrm{B}$, since it is known that any homeomorphism $f: X \rightarrow X$ satisfying the assumptions of Theorem $\mathrm{C}$ is isotopic to the identity on $D$ (see [19] for details). However, Theorem $\mathrm{C}$ better encapsulates the motivation for our results, because we formulate them for a rather wide class of continua, which may contain no arcs at all, and therefore they may admit no isotopies connecting two distinct homeomorphisms. Our first result is the following theorem.

THEOREM 1.1. Let $h: \mathbb{R}^{2} \rightarrow \mathbb{R}^{2}$ be an orientation preserving planar homeomorphism, and let $C$ be a continuum such that $h^{-1}(C) \cup C$ is acyclic. If there is a point $c \in C$ such that $\left\{h^{-i}(c): i \in \mathbb{N}\right\} \subseteq C$, or $\left\{h^{-i}(c): i \in \mathbb{N}\right\} \subseteq C$, then $C$ also contains a fixed point of $h$.

Note that Theorem 1.1 generalizes Theorem A because if $h(C)=C$ then $C \cup h^{-1}(C)=$ $C$ and $\left\{h^{-i}(c): i \in \mathbb{Z}\right\} \subseteq C$ for any $c \in C$. It is also quite clear that Theorem 1.1 implies Theorem B if $X$ is one-dimensional, because in this case $h^{-1}(C) \cup C$ is acyclic as a subcontinuum of the one-dimensional and simply connected $X$. Finally, Theorem 1.1 is false without the assumption that $h^{-1}(C) \cup C$ is acyclic. Indeed, it is enough to consider the rotation $h$ of the plane about the origin by $180^{\circ}$, and let $C$ be the upper semicircle in the unit circle. Then $C$ contains a 2-periodic orbit (the end points) but no fixed point.

At this point we would like to point out that some different fixed point results for noninvariant continua were proven in [7, Ch. 5] by Blokh et al. The authors showed that every positively oriented map of the complex plane that strongly scrambles the boundary of an acyclic (potentially non-invariant) continuum $C$ must have a fixed point in $C$, and a related result is also proven for certain maps on dendrites. Since positively oriented maps generalize orientation preserving homeomorphisms their results are related to ours, but the emphasis of their approach is more on the structure of $X$, expressed in one-step 'geometric' conditions, whereas our approach can be considered more dynamical in nature, as determined by iterations of the homeomorphism on $X$. The reader is referred to [7] for more details.

A natural question to ask next is: what if $h$ reverses orientation? Can one also generalize Bell's theorem [5] in the same way? This question seems a little bit more delicate, as we shall now explain. First, note that if $h$ reverses orientation then $h^{2}$ preserves orientation, and so it is natural to expect for $h$ either a fixed point or a point of least period 2, under assumptions similar to those of Theorem 1.1. Note also that the fact that $h^{-1}(C) \cup C$ is 
acyclic, for a continuum $C$, does not imply that $h^{-2}(C) \cup C$ is acyclic, so a straightforward application of Theorem 1.1 in the orientation reversing case does not automatically give us fixed points for $h^{2}$. Next recall that an important property of orientation preserving homeomorphisms, which is often used to prove the Cartwright-Littlewood theorem, is the result attributed to Brouwer [12]: if a planar homeomorphism has a bounded orbit then it also has a fixed point. This property is not shared by orientation reversing homeomorphisms. In 1981 Boyles [11] constructed an example of such a homeomorphism with every orbit bounded but no fixed points. Inspired by her construction, in $\S 4$ we will exhibit an example of an orientation reversing homeomorphism $H$ with every orbit bounded, and a continuum $C$, with $H^{-1}(C) \cup C$ acyclic, that contains periodic points of any even period, but contains no fixed points (in fact $H$ is fixed-point-free).

Nonetheless, one can also hope for a counterpart of Theorem 1.1 for orientation reversing homeomorphisms, if a periodic point of least period 2 in $C$ is to be guaranteed instead of a fixed point. We obtain such a result in Theorem 1.2, where we show that assuming a continuum $C$, with the property that $h^{-1}(C) \cup C$ is acyclic, contains a periodic orbit of period $k>1$, one can infer the existence of a 2-periodic point in $C$. This result does not seem to follow from Brown's work but can be proved using Bonino's powerful result on linked periodic orbits [8]. Very similar arguments were previously used by the author [9] to show that any orientation reversing planar homeomorphism with a $k$-periodic orbit $(k>1)$ contained in an invariant acyclic continuum $C$ must have a 2-periodic orbit in $C$.

THEOREM 1.2. Let $g: \mathbb{R}^{2} \rightarrow \mathbb{R}^{2}$ be an orientation reversing planar homeomorphism, and let $C$ be a continuum which contains a periodic orbit of least period $k>1$. If $g^{-1}(C) \cup C$ is acyclic then $C$ contains a point of least period 2.

Although, based on Theorem 1.1, one could expect that there must be either a fixed point or a 2-periodic point in $C$, one can see that Theorem 1.2 determines more than just a mere alternative. In particular, $C$ may or may not contain a fixed point, but it is guaranteed to contain a point of least period 2 . In the final section of our paper we show that the above result is in a sense the best possible. In particular, one can neither expect a fixed point under the assumptions, nor weaken the assumptions and require that a non-periodic orbit contained in $C$ will force a point of least period 2. Note that, as a corollary to the above two theorems, we obtain the following result.

COROLlaRY 1.3. Let $h: \mathbb{R}^{2} \rightarrow \mathbb{R}^{2}$ be a planar homeomorphism and $X$ be a onedimensional acyclic continuum. Suppose there are $n$ components of $X \cap h(X)$, each of which contains a periodic orbit of period $k>1$. Then:

(1) if $h$ preserves orientation then $h$ has at least $n$ fixed points in $X$;

(2) if $h$ reverses orientation then $h$ has at least $\lfloor(n+1) / 2\rfloor$ orbits of period 2 in $X$.

\section{Preliminaries}

Given a set $D$, we shall denote its boundary by $\partial D$. Given a homeomorphism $h: \mathbb{R}^{2} \rightarrow$ $\mathbb{R}^{2}$, we define $h^{1}(x)=h(x)$ and $h^{k+1}(x)=h \circ h^{k}(x)$ for all $k \in \mathbb{N}$ and $x \in \mathbb{R}^{2}$. Similarly, $h^{-k-1}(x)=h^{-1} \circ h^{-k}(x)$ for every $k \in \mathbb{N}$. A point $x \in \mathbb{R}^{2}$ is said to be $k$-periodic or of 
least period $k$ if $h^{k}(x)=x$ and $h^{i}(x) \neq x$ for $i=1, \ldots, k-1$. If $k=1$ (that is, $\left.h(x)=x\right)$, then we say that $x$ is a fixed point of $h$. The forward orbit of $x$ is given by $\left\{h^{n}(x): n=\right.$ $1,2,3, \ldots\}$ and the backward orbit of $x$ is given by $\left\{h^{-n}(x): n=1,2,3, \ldots\right\}$. If $x$ is $k$-periodic then the orbit of $x$ is given by $\left\{x, h(x), \ldots, h^{k-1}(x)\right\}$ and is said to be a $k$ periodic orbit. Following [8], we say that two periodic orbits $\mathcal{O}$ and $\mathcal{O}^{\prime}$ are linked if one cannot find a Jordan curve $C \subseteq \mathbb{S}^{2}$ separating $\mathcal{O}$ and $\mathcal{O}^{\prime}$ which is freely isotopic to $h(C)$ in $\mathbb{S}^{2} \backslash\left(\mathcal{O} \cup \mathcal{O}^{\prime}\right)$. $C$ and $h(C)$ are freely isotopic in $\mathbb{S}^{2} \backslash\left(\mathcal{O} \cup \mathcal{O}^{\prime}\right)$ if there is an isotopy $\left\{i_{t}: \mathbb{S}^{1} \rightarrow \mathbb{S}^{2} \backslash\left(\mathcal{O} \cup \mathcal{O}^{\prime}\right): 0 \leq t \leq 1\right\}$ from $i_{0}\left(\mathbb{S}^{1}\right)=C$ to $i_{1}\left(\mathbb{S}^{1}\right)=h(C)$; that is, $i_{t}\left(\mathbb{S}^{1}\right)$ is a Jordan curve for any $t$ ( $\mathbb{S}^{1}$ denotes the unit circle). Also, recall that if $U$ is an open surface and $(\tilde{U}, \tau)$ is its universal covering space then, given a homeomorphism $h: U \rightarrow U$, there exists a lift homeomorphism $\tilde{h}: \tilde{U} \rightarrow \tilde{U}$ such that the following diagram commutes.

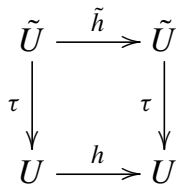

Additionally if $h(x)=y$ then $\tilde{h}$ is uniquely determined by the choice of two points $\tilde{x} \in$ $\tau^{-1}(x), \tilde{y} \in \tau^{-1}(y)$ and setting $\tilde{h}(\tilde{x})=\tilde{y}$.

\section{Proofs of the main results}

Our proof of Theorem 1.1 will follow the approach used by Brown in [13] to prove the Cartwright-Littlewood fixed point theorem. His proof by contradiction was based on an idea that an orientation preserving homeomorphism $h: \mathbb{R}^{2} \rightarrow \mathbb{R}^{2}$, with an acyclic continuum $X=h(X)$ and the fixed point set Fix $(h)$, can be lifted to a homeomorphism $\tilde{h}$ of the universal covering $(\tilde{U}, \tau)$ of a component of $\mathbb{R}^{2} \backslash \operatorname{Fix}(h)$ that will have a bounded orbit, yet no fixed point. This leads to a contradiction with Brouwer's theorem. The approach works because $\tilde{U}$ is homeomorphic to $\mathbb{R}^{2}$ and the lift $\tilde{h}$ preserves orientation. Strictly speaking, the existence of a bounded orbit of $\tilde{h}$ is guaranteed by the fact that $X$ lifts to disjoint homeomorphic copies (the components of $\left.\tau^{-1}(X)\right)$ and $\tilde{h}$ can be chosen so that one such copy is invariant. Indeed, it is well known that every acyclic continuum $X$ is the intersection of a decreasing sequence of closed disks $\left\{D_{n}: n \in \mathbb{N}\right\}$; that is, $D_{n} \subseteq D_{n-1}$ for every $n$, and $X=\bigcap_{n \in \mathbb{N}} D_{n}$. In particular, $X$ has arbitrarily small simply connected neighborhoods that can separate $X$ from $\operatorname{Fix}(h)$.

Proof of Theorem 1.1. Refer to Figure 2. By contradiction, suppose that $\operatorname{Fix}(h) \cap C=\emptyset$. Let $U$ be the component of $\mathbb{R}^{2} \backslash \operatorname{Fix}(h)$ that contains $C$. Note that $h(U)=U$, since $h$ is onto, $h(U)$ must be contained in one of the complementary domains of Fix $(h)$ and $h(U) \cap$ $U \neq \emptyset$. Let $(\tau, \tilde{U})$ be the universal cover of $U$. Note that $\tilde{U}$ is homeomorphic to $\mathbb{R}^{2}$ and $C$ lifts to disjoint homeomorphic copies in $\tilde{U}$, because of $h^{-1}(C) \cup C$ being acyclic [13]. Let $\tilde{C}$ be one such a copy.

Case 1. First suppose that $c$ has a backward orbit $\mathcal{O}=\left\{h^{-i}(c): i \in \mathbb{N}\right\}$ contained in $C$. Let $x=h^{-1}(c)$.

There is a lift $\tilde{h}: \tilde{U} \rightarrow \tilde{U}$ of $h$ determined by $\tilde{h}(\tilde{C}) \cap \tilde{C} \neq \emptyset$. Namely, let $\tilde{x} \in \tau^{-1}(x) \cap$ $\tilde{C}$. If $\tilde{y} \in \tau^{-1}(c) \cap \tilde{C}$ then the condition $\tilde{h}(\tilde{x})=\tilde{y}$ uniquely determines the lift $\tilde{h}$. 


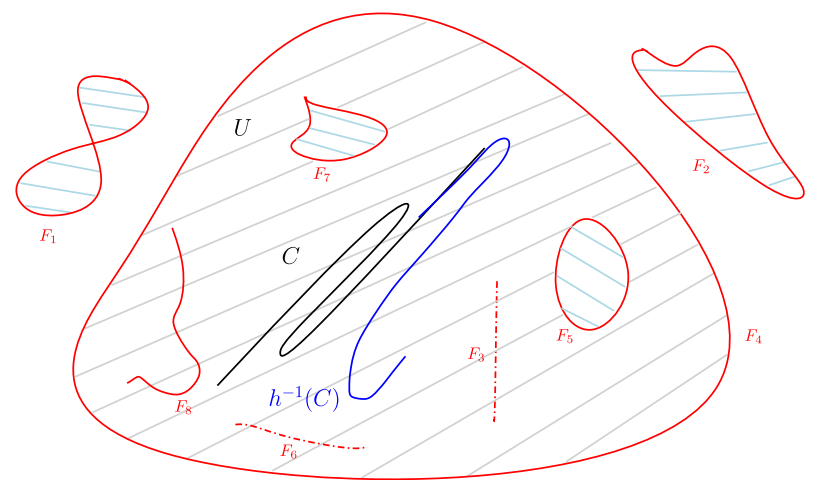

FIGURE 2. $U$ is the complementary domain of Fix $(h)=\bigcup_{i=1}^{8} F_{i}$ that contains $C \cup h^{-1}(C)$.

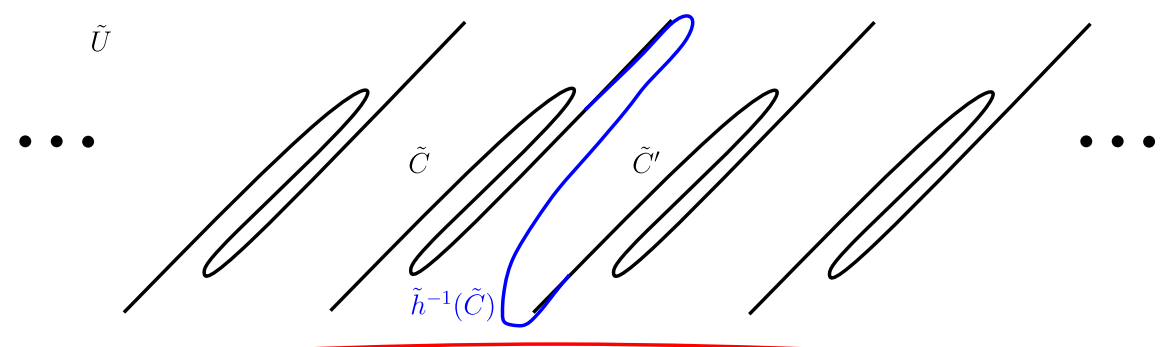

FIGURE 3. Proof of Claim 3.2: $\tilde{h}^{-1}(\tilde{C})$ cannot intersect $\tilde{C}^{\prime}$.

Claim 3.1. $\tilde{h}^{-1}(\tilde{x}) \in \tilde{C}$.

Proof of Claim 3.1. Refer to Figure 3. We shall use the fact that, under the covering map $\tau$, continua which map to acyclic continua must map one-to-one. Set $Q=h^{-1}(C)$ and let $\tilde{Q}$ be the component of $\tau^{-1}(Q)$ such that $\tilde{Q}=\tilde{h}^{-1}(\tilde{C})$. By contradiction, suppose $\tilde{h}^{-1}(\tilde{x}) \notin \tilde{C}$. Then there is another copy $\tilde{C}^{\prime}$ of $C$ in $\tau^{-1}(C)$ such that $\tilde{h}^{-1}(\tilde{x}) \in \tilde{C}^{\prime}$. Note that $\tilde{h}^{-1}(\tilde{x}) \in \tilde{Q} \cap \tilde{C}^{\prime}$ and $\tilde{x} \in \tilde{Q} \cap \tilde{C}$. But then $\tilde{Q} \cup \tilde{C} \cup \tilde{C}^{\prime}$ is a continuum with $\tau\left(\tilde{Q} \cup \tilde{C} \cup \tilde{C}^{\prime}\right)=C \cup h^{-1}(C)$, and $C \cup h^{-1}(C)$ is acyclic so $\tau^{-1}\left(C \cup h^{-1}(C)\right)$ cannot contain two components of $\tau^{-1}(C)$. This leads to a contradiction.

Claim 3.2. $\tilde{h}^{-i}(\tilde{x}) \in \tilde{C}$ for every $i$.

Proof of Claim 3.2. This follows by induction. Suppose that we have already proved that $\tilde{h}^{-i}(\tilde{x}) \in \tilde{C}$ for all $i=1, \ldots, j$. Now replace $\tilde{x}$ with $\tilde{h}^{-j}(\tilde{x})$ in the proof of the above claim and repeat the same arguments. It follows that $\tilde{h}^{-j-1}(\tilde{x}) \in \tilde{C}$ and then, by induction, that $\tilde{h}^{-i}(\tilde{x}) \in \tilde{C}$ for every $i$.

To complete the proof of case 1 note that $\tilde{h}^{-1}: \tilde{U} \rightarrow \tilde{U}$ is a planar homeomorphism with $\left\{\tilde{h}^{-i}(\tilde{x}): i \in \mathbb{N}\right\} \subseteq \tilde{C}$. Since $\tilde{h}^{-1}$ has no fixed points, we have obtained a contradiction with Brouwer's theorem [12]. 
Case 2. Now suppose that $c$ has a forward orbit $\mathcal{O}^{\prime}=\left\{h^{i}(c): i \in \mathbb{N}\right\}$ contained in $C$. Then the proof of case 1 can be repeated because the fact that $h$ is a homeomorphism and $h^{-1}(C) \cup C$ is acyclic implies that $C \cup h(C)$ is acyclic as well.

Now we shall prove Theorem 1.2. Our proof, similar to [9, proof of Theorem 3.1], will rely on the powerful result of Bonino on linked periodic orbits [8]. Recall that two periodic orbits $\mathcal{O}$ and $\mathcal{O}^{\prime}$ of a homeomorphism $h$ are linked if one cannot find a Jordan curve $C \subseteq \mathbb{S}^{2}$ separating $\mathcal{O}$ and $\mathcal{O}^{\prime}$ which is freely isotopic to $h(C)$ in $\mathbb{S}^{2} \backslash\left(\mathcal{O} \cup \mathcal{O}^{\prime}\right)$. $C$ and $h(C)$ are freely isotopic in $\mathbb{S}^{2} \backslash\left(\mathcal{O} \cup \mathcal{O}^{\prime}\right)$ if there is an isotopy $\left\{i_{t}: \mathbb{S}^{1} \rightarrow \mathbb{S}^{2} \backslash\left(\mathcal{O} \cup \mathcal{O}^{\prime}\right): 0 \leq t \leq 1\right\}$ from $i_{0}\left(\mathbb{S}^{1}\right)=C$ to $i_{1}\left(\mathbb{S}^{1}\right)=h(C)$; that is, $i_{t}\left(\mathbb{S}^{1}\right)$ is a Jordan curve for any $t$.

Proof of Theorem 1.2. We shall show that, under the assumptions, if $C$ contains a $k$ periodic $(k>1)$ orbit $\mathcal{O}$ then the 2-periodic orbit linked to $\mathcal{O}$, guaranteed by [8], must intersect $C$. Note that if $k=2$ then there is nothing to prove, so assume $k>2$. Compactify $\mathbb{R}^{2}$ by a point $\infty$ to obtain $\mathbb{S}^{2}=\mathbb{R}^{2} \cup\{\infty\}$. Note that $g: \mathbb{R}^{2} \rightarrow \mathbb{R}^{2}$ can be extended to a homeomorphism $\tilde{g}: \mathbb{S}^{2} \rightarrow \mathbb{S}^{2}$ by setting $\tilde{g} \mid \mathbb{R}^{2}=g$, and $\tilde{g}(\infty)=\infty . g$ and $\tilde{g}$ have exactly the same $k$-periodic points for any $k>1$. Let $\mathcal{O}$ be the $k$-periodic orbit contained in $C$. By Bonino's result there is a 2-periodic orbit $\mathcal{O}^{\prime} \subseteq \mathbb{S}^{2}$ that is linked to $\mathcal{O}$. We will show that $\mathcal{O}^{\prime} \cap C \neq \emptyset$. This will imply that $\mathcal{O}^{\prime} \subseteq g^{-1}(C) \cup C$.

Refer to Figure 4. By contradiction, suppose that $\mathcal{O}^{\prime} \cap C=\emptyset$. Then also $\mathcal{O}^{\prime} \cap$ $\tilde{g}^{-1}(C)=\emptyset$. Consequently, $\mathcal{O}^{\prime} \subseteq U$ for the complementary domain $U$ of $C$. Since $C \cup \tilde{g}^{-1}(C)$ is acyclic, it is the intersection of a family of closed disks with diameters decreasing to 0 , and there is a Jordan curve $S \subseteq \mathbb{S}^{2}$ separating $\mathcal{O}^{\prime}$ from $C \cup \tilde{g}^{-1}(C)$. Let $U_{\infty}$ and $U_{b}$ be the two complementary domains of $S$, with $\infty \in U_{\infty}$. Then $C \cup \tilde{g}^{-1}(C)$ is contained in $U_{b}$ and $\mathcal{O}^{\prime} \subseteq U_{\infty}$. Note that $\tilde{g}(S)$ is a Jordan curve that bounds $C$ (therefore also $\mathcal{O})$, and $\mathcal{O}^{\prime}$ is contained in $\tilde{g}\left(U_{\infty}\right)$, as $\tilde{g}\left(\mathcal{O}^{\prime} \cup\{\infty\}\right)=\mathcal{O}^{\prime} \cup\{\infty\}$. Let $D$ be a disk neighborhood of $\infty$ in $\mathbb{S}^{2}$ such that $\mathcal{O}^{\prime} \subseteq D$ and $D$ is disjoint with $S \cup \tilde{g}(S)$. D can be contracted to the point $\infty$. Then $\mathbb{S}^{2} \backslash\left(C \cup g^{-1}(C) \cup\{\infty\}\right)$ is an open annulus that contains $S$ and $\tilde{g}(S)$ as essential Jordan curves. Therefore $S$ and $\tilde{g}(S)$ are freely isotopic in $\mathbb{S}^{2} \backslash(C \cup\{\infty\})$ and consequently in $\mathbb{S}^{2} \backslash\left(\mathcal{O} \cup \mathcal{O}^{\prime}\right)$. But this means that $\mathcal{O}$ and $\mathcal{O}^{\prime}$ are not linked, leading to a contradiction.

Finally we derive Corollary 1.3.

Proof of Corollary 1.3. Let $C$ be a component of $h(X) \cap X$. First, note that $h^{-1}(C) \cup C$ is an acyclic continuum by one-dimensionality of $X$ and the fact that $h^{-1}(C) \cup C \subseteq X$. To prove (1) simply notice that, by Theorem $1.1, h$ will have a fixed point in each component of $X \cap h(X)$ that contains a periodic orbit. Since there are $n$ such components, we deduce that there must be at least $n$ fixed points.

To prove (2) suppose that $C_{1}, C_{2}$ and $C_{3}$ are three components of $X \cap h(X)$ and $\mathcal{O}_{1}, \mathcal{O}_{2}$ and $\mathcal{O}_{3}$ are $k$-periodic orbits $(k>2)$ such that $\mathcal{O}_{i} \subseteq C_{i}$ for $i=1,2,3$. By Theorem 1.2 there are 2-periodic orbits $\mathcal{O}_{1}^{\prime}, \mathcal{O}_{2}^{\prime}$ and $\mathcal{O}_{3}^{\prime}$ such that $C_{i} \cap \mathcal{O}_{i}^{\prime} \neq \emptyset$ for $i=1,2,3$. It suffices to show that $\mathcal{O}_{i}^{\prime} \neq \mathcal{O}_{j}^{\prime}$ for some $i, j \in\{1,2,3\}$. By contradiction, suppose that $\mathcal{O}_{1}^{\prime}=\mathcal{O}_{2}^{\prime}=\mathcal{O}_{3}^{\prime}$. Let $p \in \mathcal{O}_{1}^{\prime} \cap C_{1}$. Then $p \notin C_{2} \cup C_{3}$. Therefore $h(p) \in C_{2} \cap C_{3}$. We obtain a contradiction since $C_{2}$ and $C_{3}$ are disjoint components of $X \cap h(X)$. 


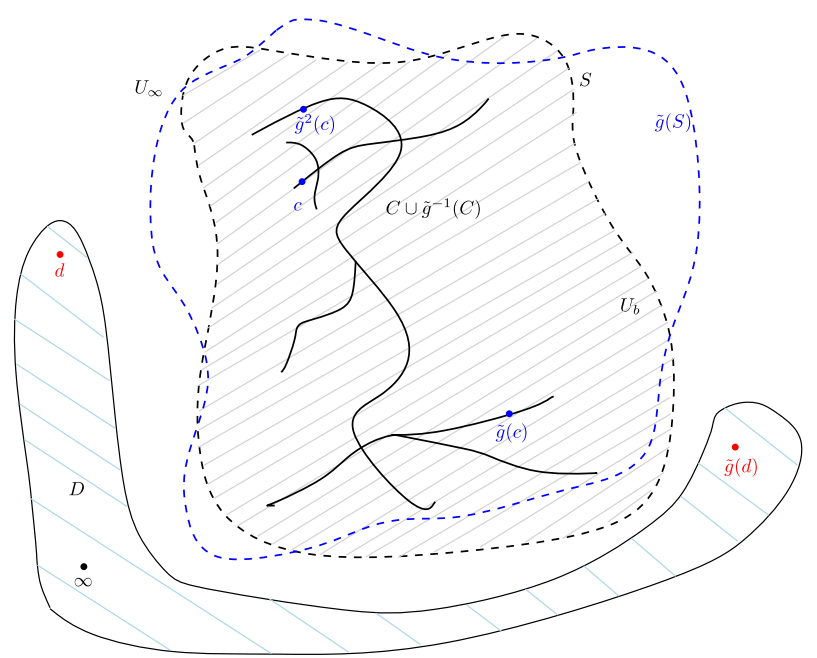

FIGURE 4. Proof of Theorem 1.2 with $\mathcal{O}=\left\{c, \tilde{g}(c), \tilde{g}^{2}(c)\right\}$ and $\mathcal{O}^{\prime}=\{d, \tilde{g}(d)\}$.

\section{Examples}

In this section we discuss restrictions on potential generalizations of our results. We shall show that in Theorem 1.2 it is not enough to require that $C$ contains an infinite (nonperiodic) orbit, nor can one, under the assumptions of Theorem 1.2, infer the existence of a fixed point.

THEOREM 4.1. There exist an orientation reversing homeomorphism $H$ and a continuum C such that:

(1) $H^{-1}(C) \cup C$ is an acyclic continuum and contains a $2 k$-periodic orbit for every $k \geq 1$.

(2) All orbits of $H$ are bounded.

(3) $H$ has no fixed points.

Proof. Refer to Figure 5. Let $D=\left\{\left(x_{1}, x_{2}\right) \mid x_{1}^{2}+x_{2}^{2} \leq 1\right\}$. Consider a homeomorphism $\phi: D \rightarrow D$ that has periodic orbits of all periods. (One such example can be easily obtained by [2] and [3] from the tent map $f$ on the unit interval. The inverse limit $Y_{f}=$

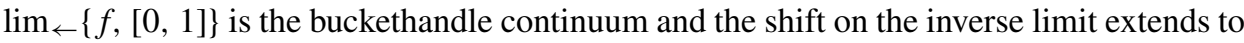
a disk homeomorphism with $Y_{f}$ as a chaotic attractor and all periods present.) One can also easily ensure that $\phi(x)=x$ for every $x \in \partial D$. Let $D_{1}=\left\{\left(x_{1}, x_{2}\right) \mid\left(x_{1}-2\right)^{2}+x_{2}^{2} \leq 1\right\}$ and $D_{-1}=\left\{\left(x_{1}, x_{2}\right) \mid\left(x_{1}+2\right)^{2}+x_{2}^{2} \leq 1\right\}$. Let $g\left(x_{1}, x_{2}\right)=\phi\left(x_{1}-2, x_{2}\right)$ for $\left(x_{1}, x_{2}\right) \in$ $D_{1}, g\left(x_{1}, x_{2}\right)=\phi\left(-x_{1}+2, x_{2}\right)$ for $\left(x_{1}, x_{2}\right) \in D_{-1}$ and $g(x)=x$ if $x \notin\left(D_{1} \cup D_{-1}\right)$. Set $h=g \circ r$, where $r$ is the reflection about the $y$-axis. Set $E=\left\{\left(x_{1}, x_{2}\right) \mid x_{1}^{2}+4 x_{2}^{2} \leq 1\right\}$. Consider the continuum $X=D_{1} \cup D_{-1} \cup E$. To obtain the desired homeomorphism $H$, one can easily modify $h$ in the infinite strip $S=\left\{\left(x_{1}, x_{2}\right):\left|x_{1}\right|<1\right\}$, without any changes in the complement of $S$, so that every point in $S$ is moved slightly upward, yet $H^{-1}(C) \cup C$ is acyclic (note that $H(C) \cap C$ contains the 2-periodic orbit $\{(-1,0),(1,0)\}$ ). In fact this last modification can be obtained using Boyles' homeomorphism from [11] (since in her example all points outside of $S$ are 2-periodic), which will guarantee that all orbits are 


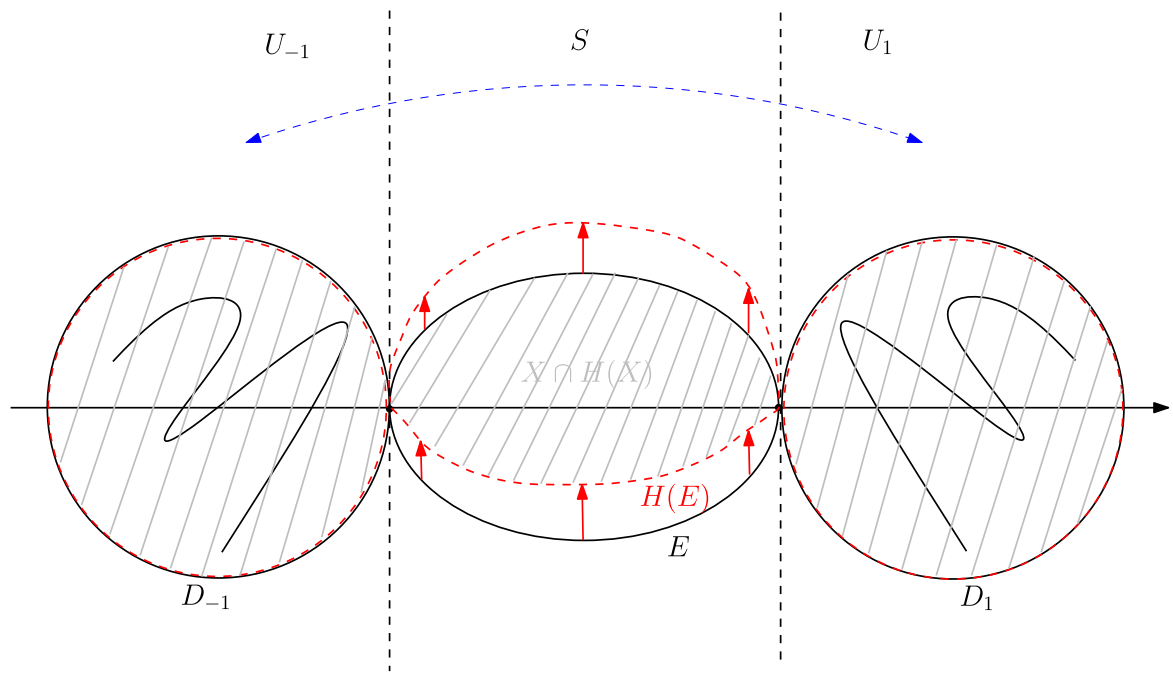

FIGURE 5. The construction of the orientation reversing homeomorphism $H$.

bounded, as promised. Clearly $H$ has no fixed points, as all points in $S$ are moved and $\mathbb{R}^{2} \backslash S$ consists of two 2-periodic disjoint closed regions $U_{-1}$ and $U_{1}$; that is, $H\left(U_{-1}\right)=U_{1}$ and $H\left(U_{1}\right)=U_{-1}$.

Example 4.2. There exist an orientation reversing homeomorphism $h: \mathbb{R}^{2} \rightarrow \mathbb{R}^{2}$ and a continuum $C$ such that $h^{-1}(C) \cup C$ is an acyclic continuum and contains an infinite (nonperiodic) orbit of $h$, but $h$ has no points of least period 2 .

Proof. Consider the orientation reversing planar homeomorphism given by $h(x, y)$ $=(-x-x|x|, y+y|1-y|)$. Then the $\operatorname{arc} C=\{(x, y): x=0, y \in[0,1]\}$ is invariant under $h$, any $c \in C \backslash\{(0,0),(0,1)\}$ has an infinite (non-periodic) orbit in $C$, but there are no points of least period 2 for $h$.

Note that the above example shows that in the assumptions of Bonino's result [8] one needs a $k$-periodic orbit $(k>1)$, and non-periodic orbits do not force linked 2-periodic orbits. We conclude with the following question.

Question. Suppose that $h: \mathbb{R}^{2} \rightarrow \mathbb{R}^{2}$ is an orientation preserving planar homeomorphism and $X$ is an acyclic continuum. Let $C$ be a component of $X \cap h(X)$. Is there is a $c \in C$ such that $\left\{h^{-i}(c): i \in \mathbb{N}\right\} \subseteq C$ or $\left\{h^{-i}(c): i \in \mathbb{N}\right\} \subseteq C$, must $C$ also contain a fixed point of $h$ ?

Acknowledgement. The author is indebted to the referee for careful reading of this paper, insightful comments and the many invaluable suggestions that greatly helped to improve the paper. This work was supported by the European Regional Development Fund in the IT4Innovations Centre of Excellence project (CZ.1.05/1.1.00/02.0070). Furthermore, the author gratefully acknowledges partial support from the MSK DT1 Support of Science and Research in the Moravian-Silesian Region 2013 and 2014 (RRC/05/2013) and (RRC/07/2014). 


\section{REFERENCES}

[1] J. M. Aarts and R. J. Fokkink. Fixed points of the bucket handle. Proc. Amer. Math. Soc. 126(3) (1998), $881-885$.

[2] M. Barge and J. Martin. Chaos, periodicity, and snakelike continua. Trans. Amer. Math. Soc. 289 (1985), 355-363.

[3] M. Barge and J. Martin. The construction of global attractors. Proc. Amer. Math. Soc. 110(2) (1990), $52-525$.

[4] M. Barge and R. M. Gillette. Indecomposability and dynamics of invariant plane separating continua. Continuum Theory and Dynamical Systems (Arcata, CA, 1989) (Contemporary Mathematics, 117). American Mathematical Society, Providence, RI, 1991, pp. 13-38.

[5] H. Bell. A fixed point theorem for plane homeomorphism. Fund. Math. 100 (1978), 119-128.

[6] R. H. Bing. A homogeneous indecomposable plane continuum. Duke Math. J. 15 (1948), 729-742.

[7] A. M. Blokh, R. J. Fokkink, J. C. Mayer, L. G. Oversteegen and E. D. Tymchatyn. Fixed point theorems in plane continua with applications. Mem. Amer. Math. Soc. 224 (2013).

[8] M. Bonino. Nielsen theory and linked periodic orbits of homeomorphisms of $\mathbb{S}^{2}$. Math. Proc. Cambridge Philos. Soc. 140(3) (2006), 425-430.

[9] J. P. Boroński. Fixed points and periodic points of orientation-reversing planar homeomorphisms. Proc. Amer. Math. Soc. 138 (2010), 3717-3722.

[10] J. P. Boroński and P. Oprocha. On indecomposability in chaotic attractors. Proc. Amer. Math. Soc. 143(8) (2015), 3659-3670.

[11] S. M. Boyles. A counterexample to the bounded orbit conjecture. Trans. Amer. Math. Soc. 266(2) (1981), 415-422.

[12] L. E. Brouwer. Beweis des ebenen Translationssatzes. Math. Ann. 72 (1912), 36-54.

[13] M. Brown. A short short proof of the Cartwright-Littlewood theorem. Proc. Amer. Math. Soc. 65(2) (1977), 372.

[14] M. L. Cartwright and J. E. Littlewood. Some fixed point theorems. Ann. of Math. (2) 54 (1951), 1-37, with appendix by H. D. Ursell.

[15] O. H. Hamilton. A short proof of the Cartwright-Littlewood fixed point theorem. Canad. J. Math. 6 (1954), 522-524.

[16] K. Kuperberg. Fixed points of orientation reversing homeomorphisms of the plane. Proc. Amer. Math. Soc. 112(1) (1991), 223-229.

[17] K. Kuperberg. A lower bound for the number of fixed points of orientation reversing homeomorphisms. The Geometry of Hamiltonian Systems (Berkeley, CA, 1989) (Mathematical Sciences Research Institute Publications, 22). Springer, New York, 1991, pp. 367-371.

[18] R. D. Mauldin. The Scottish Book, 2nd edn. Birkhäuser, Basel, 2015.

[19] G. Ostrovski. Fixed point theorem for non-self maps of regions in the plane. Topology Appl. 160(7) (2013), 915-923.

[20] V. A. Pliss. Certain questions of behavior of solutions of a periodic dissipative system of the second order. Differ. Uravn. 2 (1966), 723-735.

[21] M. Shub. What is a horseshoe? Notices Amer. Math. Soc. 52(5) (2005), 516-517. 